\title{
Gait speed may predict development of Type 2 diabetes: A pilot study among elderly Japanese
}

\author{
Shuhei Nakanishi ${ }^{{ }^{*}}$, Gen Takezawa², Yasuyuki Taooka ${ }^{3}$, Katsuko Kikkawa $^{4}$, \\ Naoko Matsumoto ${ }^{5}$, Toru Hidaka ${ }^{4}$ \\ ${ }^{1}$ Department of Molecular and Internal Medicine, Graduate School of Biomedical \& Health Sciences, Hiroshima University, \\ Hiroshima, Japan; *Corresponding Author: nshuhei@hiroshima-u.ac.jp \\ ${ }^{2}$ Department of Surgery, Aki-Ota Hospital, Hiroshima, Japan \\ ${ }^{3}$ Department of Internal Medicine, Aki-Ota Hospital, Hiroshima, Japan \\ ${ }^{4}$ Welfare Division, Health and Social Welfare Department, Aki-Ota Town Government, Hiroshima, Japan \\ ${ }^{5}$ Fitness Club Company, Hiroshima, Japan
}

Received 24 December 2013; revised 18 January 2014; accepted 26 January 2014

Copyright (C) 2014 Shuhei Nakanishi et al. This is an open access article distributed under the Creative Commons Attribution License, which permits unrestricted use, distribution, and reproduction in any medium, provided the original work is properly cited. In accordance of the Creative Commons Attribution License all Copyrights (C) 2014 are reserved for SCIRP and the owner of the intellectual property Shuhei Nakanishi et al. All Copyright (C) 2014 are guarded by law and by SCIRP as a guardian.

\section{ABSTRACT}

Aims: Epidemiologic evidence suggests that physical activity is associated with reduced risk of Type 2 diabetes. Walking is simple, effective, and by far the most prevalent physical activity among older adults. However, it is difficult to recognize and evaluate performance-based parameters of physical activity. The purpose of this study was to evaluate the importance of performance-based gait speed among several physical measurements in the development of Type 2 diabetes using long-term community-based data on elderly Japanese persons. Methods: 10 baseline physical measurements, including habitual (HGS) and fast (FGS) gait speed, were examined in 102 elderly Japanese persons living independently in the community (mean age: 71.1 years old) who were not previously diagnosed with diabetes. The subjects then participated in a daily exercise program consisting of walking, stretching, muscle strengthening, and balance exercises and were followed for an average of 4.16 years. Using Cox proportional hazards models with adjustment for age, sex, and category of fasting glucose status, we investigated whether gait speed and/or other physical measurements are associated with development of Type 2 diabetes. Results: 9 subjects developed Type 2 diabetes. Among the 10 physical parameters examined, prolongation of HGS and FGS per second was the only statistically significant factors, with hazard ratios $1.83(1.15-2.89, \mathrm{P}=$ $0.010)$ and $2.93(1.43-6.03, P=0.003)$, respectively. Conclusions: We found a negative association between the development of Type 2 diabetes and gait speed among elderly Japanese people. We conclude that encouraging physical activity and preserving walking capacity may be beneficial for preventing Type 2 diabetes.

\section{KEYWORDS}

Gait Speed; Type 2 Diabetes; Japanese Elderly Population

\section{INTRODUCTION}

Type 2 diabetes mellitus is a growing public health concern worldwide, with the number of people developing the condition increasing rapidly [1]. The estimated prevalence of suspected diabetes mellitus case numbers among adults in the world, the United States, and Japan was about 366 million, 23.7 million, and 10.7 million, respectively, in 2011, and is expected to reach about 552 million in the world in 2030 [2]. Because prevalence of diabetes mellitus increases with age, individuals over age 60 with diabetes mellitus now exceed two-thirds of the total number of patients with Type 2 diabetes in Japan, where the society is rapidly aging [3]. Accordingly, preventing elderly people from developing Type 2 diabetes is an urgent public health need among Japan's aging population.

Epidemiologic evidence suggests that physical activity 
is associated with reduced risk of Type 2 diabetes. Walking is simple, effective, and by far the most common physical activity among older adults, for whom the exercise is feasible, accessible, and relatively safe [4]. In a large cohort study, $\mathrm{Hu}$ et al. found a strong association between walking pace assessed by questionnaire and risk of Type 2 diabetes even after adjustment for several known confounders [5]. However, it is not often in a clinical setting that measurement takes place of performance-based parameters of physical activity, such as gait speed. Level of physical activity was assessed using questionnaires instead of directly measuring perfor-mance-based physical activity in several studies. In addition, although there exist several methods other than gait speed for evaluating physical activity, studies examining the association between development of Type 2 diabetes and performance-based parameters of physical activity are relatively sparse.

The Aki-Ota region is a rural area in the northern part of Hiroshima Prefecture in Japan, with little population movement and an aged population of 7308 persons, where around $45 \%$ of the population were 65 years of age or more as of November, 2012. The Health-up Model Project, scheduled by the All Japan Federation of National Health Insurance Organizations, began in 2001 in this region [6]. A primary purpose of the project was to prevent adult disease by promoting physical exercise. Even after the project concluded, public health nurses in the Aki-Ota region regularly encouraged the residents to exercise with the help of health and fitness trainers using school gymnasiums or civic halls to maintain good exercise habits. In particular, in some elderly persons, performance-based physical activity was evaluated using several measurements, including gait speed, before exercise began. We hypothesized that, among several measurements of performance-based physical activity, gait speed may be a reliable indicator of risk of developing Type 2 diabetes in community-dwelling elderly without diabetes. We sought to test the hypothesis by analyzing the data from the Aki-Ota region.

\section{METODS}

\subsection{Study Participants and Measurements}

We recruited 35 men and 67 women aged 60 years or more who were in good health, living independently in the community, and self-reliant in daily activities, and not previously diagnosed with diabetes. They attended an exercise program, which was held 2 days per month regularly from 2002 to 2011 by the Aki-Ota public health center, on at least 2 occasions. Recruitment was announced in a public-information magazine published by the Health and Social Welfare Department of the government offices of Aki-Ota town. All subjects provided blood samples each year after an overnight fast for measurement of biochemical parameters. We used an automated enzymatic procedure to determine serum triglycerides, total cholesterol, HDL cholesterol, and LDL cholesterol levels. Concentration of blood glucose was determined with the hexokinaseglucose-6-phosphate dehydrogenase method. Glycosylated hemoglobin (HbA1c) (\%) was measured using ion-exchange high-performance liquid chromatography. HbA1c was estimated using a method equivalent to the National Glycohemoglobin Standardization Program calculated as: HbA1c (\%) = HbA1c (Japan Diabetes Society, \%) + $0.4 \%$, considering the relational expression of HbA1c measured by standard laboratory methods and the previous Japanese standards [7]. Blood pressure (mmHg) was measured by a standard mercury sphygmomanometer with subjects in the sitting position. Standing height $(\mathrm{cm})$ and body weight $(\mathrm{kg})$ were also measured at every visit. All participants' HbA1c were confirmed to be less than $6.5 \%$ at baseline. Participation in this study was strictly voluntary, and informed consent was obtained. This study was approved by the ethics committee of Hiroshima University.

\subsection{Measurements of Physical Performance}

At the beginning of every exercise session, participants received a private physical interview that included information on past and present medical history and use of medication. Subsequently, 10 measurements were carried out, as follows: Standing on one leg (seconds) [8], reaching forward with right or left outstretched arm while standing (functional reach, \% height) [8,9], turning 360 degrees from right or left side (seconds) [8], stool stepping (seconds) [8], and tandem stance test (seconds) $[8,10]$, which all are measures that can be quantified using the Berg Balance Scale (BBS) [8]. In addition, data were collected on right and left grip strength $(\mathrm{kg})$ using a dynamometer [11]. The Timed Up \& Go Test (TUG) (seconds) measured the time it took a subject to stand up from an armchair, walk a distance of $3 \mathrm{~m}$, turn, walk back to the chair, and sit down $[12,13]$. Maximal step length was considered as the vertical distance between the tip of the large toe of one foot and that of the opposite foot [14]. Functional reach and maximal step length were normalized to the height of the participant. To measure habitual and fast-speed walking (HGS and FGS, seconds) $[12,14,15]$, a 10-meter-long track was set up in each location where exercise sessions were held. HGS and FGS tests were performed at the participant's usual pace and with quick steps without acceleration and deceleration, respectively. Use of a walker or cane was allowed if needed. Gait velocity was calculated as walking distance (meters) divided by time (seconds). The ceiling performance of both tandem stance and standing on one 
leg was 30 seconds. All times were measured using a hand-held stopwatch. One health-fitness trainer continuously instructed subjects throughout the fitness training. During each exercise session, muscle-strength training using one's own weight in the sitting or standing position, stretching, training for the keeping of balance, and other such exercises were performed for a total of 90 minutes. The physical activity level of the training was calculated by an estimate of the metabolic equivalent of that activity (MET) and was a sum of the targeted 3 METs for all activities performed, including at least 10 minutes of stretching and 30 minutes of aerobic exercise. In addition, participants were encouraged by public nurses and the health trainer to walk and exercise outside for more than 30 minutes every day. This Health-up model project is described in detail elsewhere [6].

Prevalent diabetes was defined as self-report of physician diagnosis or use of hypoglycemic medication. Among participants without diabetes, presence of incident diabetes and impaired fasting glucose were identified by fasting plasma glucose level equal to or more than 126 $\mathrm{mg} / \mathrm{dL}$ and equal to or more than $110 \mathrm{mg} / \mathrm{dL}$ and less than $126 \mathrm{mg} / \mathrm{dl}$, respectively, based on the American Diabetes Association 1997 criteria [16]. The relationship between the 10 physical measurements and development of diabetes during the follow-up period was analyzed statistically as described below.

\subsection{Statistical Analysis}

Results are expressed as mean and standard deviation. Because body mass index (BMI) data did not appear to be normally distributed, they were analyzed after logarithmic transformation. Relationships between categorical variables were evaluated using $\chi^{2}$ tests. Grip strength, standing on one leg, functional reach, and turning 360 degrees were analyzed using the sum of the values for right and left extremities. Cox proportional hazards models were used to test the significance of each physical performance parameter, HbA1c, criteria of IFG, and BMI as predictors of incidence of Type 2 diabetes. In addition, to test the significance of gait speed as a predictor of Type 2 diabetes incidence, HGS and FGS were divided into quartiles based on the population (HGS, $<5.99$, 5.99 - 6.74, $6.75-7.58$, and $>7.58$ seconds; FGS, $<4.84,4.84$ - 5.35, 5.36 - 6.10, and $>6.10$ seconds); quartile-specific hazard ratios were estimated through the use of indicator variables in Cox proportional hazards models. With respect to potential confounders, we used age as a continuous value. Adjustments were made for sex and fasting glucose status as categorical values, except when the risks for the HbA1c, BMI, and category of impaired fasting glucose (IFG) were analyzed. The proportional hazards assumption was verified by inspection of log-log survival curves and by examination of Schoen- feld's partial residuals [17]. All analyses were performed with SAS version 8.2 (SAS Institute, Cary, NC).

\section{RESULTS}

Clinical characteristics of the study subjects at baseline are shown in Table 1. Mean age and follow-up period were 71.1 (SD 4.4) years and 4.16 (SD 3.35) years, respectively. Subjects with normal (NFG) and IFG numbered 83 and 19, respectively. 9 cases of incident Type 2 diabetes were registered during the follow-up period. Hazard ratios adjusted for age and sex per unit BMI and HbA1c or category of IFG were 0.87 (95\% CI: 0.67 1.14, $\mathrm{P}=0.308)$, 2.89 (1.74 - 4.80, $\mathrm{P}<0.001)$, and 5.46 (1.45 - 20.53, P $=0.012)$, respectively. Among the 10 measured physical parameters, only HGS and FGS were significantly related to development of Type 2 diabetes after adjustment for age, sex, and fasting glucose status, with hazard ratios $1.83(1.15-2.89, \mathrm{P}=0.010)$ and 2.93 (1.43 - 6.03, $\mathrm{P}=0.003$ ), respectively (Table 2$)$. In the model with adjustment for age, sex, and fasting glucose status, hazard ratios for developing diabetes by increasing quartiles of HGS were $6.35(0.50-79.99, \mathrm{P}=0.153)$, $6.61(0.48-90.73, \mathrm{P}=0.158)$, and $8.46(0.42-172.53$, P $=0.165)$, respectively $(\mathrm{P}=0.212$ for trend) compared with the lowest quartile. For increasing quartiles of FGS

Table 1. Clinical characteristics of study subjects at baseline.

\begin{tabular}{|c|c|}
\hline Male/Female & $35 / 67$ \\
\hline NFG/IFG & $83 / 19$ \\
\hline Age & $71.1 \pm 4.4$ \\
\hline Body Mass Index $\left(\mathrm{kg} / \mathrm{m}^{2}\right)$ & $24.2 \pm 2.9$ \\
\hline Systolic blood pressure (mmHg) & $149 \pm 22$ \\
\hline Diastolic blood pressure (mmHg) & $77 \pm 13$ \\
\hline Fasting glucose (mg/dl) & $101.4 \pm 9.6$ \\
\hline Total cholesterol (mg/dl) & $202 \pm 31$ \\
\hline HDL cholesterol (mg/dl) & $62 \pm 15$ \\
\hline Triglycerides (mg/dl) & $93 \pm 43$ \\
\hline HbA1c (\%) & $5.34 \pm 0.44$ \\
\hline Grip strength (L/R) (kg) & $23.4 \pm 7.3 / 25.0 \pm 6.9$ \\
\hline Functional reach (L/R) (\% height) & $22.7 \pm 4.2 / 22.3 \pm 4.0$ \\
\hline Tandem stance test (seconds) & $27.2 \pm 6.7$ \\
\hline Standing on a leg (L/R) (seconds) & $19.7 \pm 11.1 / 20.2 \pm 10.9$ \\
\hline Maximal step length (L/R) (\% height) & $63.7 \pm 10.5 / 63.2 \pm 9.5$ \\
\hline Turning 360 degrees (L/R) (seconds) & $2.53 \pm 0.66 / 2.60 \pm 0.73$ \\
\hline Stool stepping (seconds) & $8.83 \pm 2.17$ \\
\hline Habitual gait speed (seconds) & $6.90 \pm 1.33$ \\
\hline Fast gait speed (seconds) & $5.56 \pm 0.97$ \\
\hline Timed Up and Go Test (seconds) & $7.25 \pm 1.36$ \\
\hline
\end{tabular}

Data are expressed as numbers or mean \pm SD. NFG: Normal fasting glucose, IFG: Impaired fasting glucose. L: left, R: right. 
Table 2. Hazard ratios for various physical measurements.

\begin{tabular}{lcc}
\hline & Hazard ratio & $\mathrm{P}$ \\
\hline Grip strength (per kg) & $0.947(0.871-1.029)$ & 0.200 \\
Tandem stance test (per second) & $1.040(0.933-1.158)$ & 0.483 \\
Fast gait speed (per second) & $2.933(1.428-6.025)$ & 0.003 \\
Habitual gait speed (per second) & $1.826(1.154-2.889)$ & 0.010 \\
Stool stepping (per second) & $1.011(0.752-1.358)$ & 0.943 \\
Timed Up and Go Test (per second) & $1.345(0.779-2.323)$ & 0.288 \\
Functional reach (per \%) & $0.935(0.843-1.038)$ & 0.208 \\
Standing on a leg (per second) & $1.000(0.962-1.039)$ & 0.983 \\
Maximal step length (per \%) & $0.982(0.943-1.023)$ & 0.378 \\
Turning 360 degrees (per second) & $1.322(0.847-2.062)$ & 0.219 \\
Body Mass Index (per unit) & $0.021(0.000-10.386)$ & 0.222 \\
HbA1c (per \%) & $2.427(1.386-4.250)$ & 0.002 \\
IFG (vs. NFG) & $5.455(1.450-20.526)$ & 0.012 \\
\hline
\end{tabular}

Data are expressed as hazard ratio and (95\% CI) after adjustment for age, sex, and fasting glucose status. NFG: Normal fasting glucose, IFG: Impaired fasting glucose.

hazard ratios were $3.29(0.17-62.15, \mathrm{P}=0.428), 12.80$ (1.06 - 154.08, $\mathrm{P}=0.045)$, and 17.25 (0.89 - 334.26, $\mathrm{P}=$ $0.060)$, respectively ( $\mathrm{P}=0.025$ for trend) compared with the lowest quartile.

\section{DISCUSSION}

Among community-dwelling elderly subjects without diabetes, there was an inverse association between development of Type 2 diabetes and gait speed that was independent of age, sex, and fasting glucose status. Our findings support and extend previous studies that showed a strong association between low physical activity and risk of Type 2 diabetes. To our knowledge, this is the first report to describe an association between development of Type 2 diabetes and several performance-based physical parameters among elderly people.

Muscle mass tends to decline progressively with increasing age, decreased functional capacity, decreased resting metabolic rate, increased adiposity, and increased insulin resistance $[18,19]$. We found a linear relationship with no threshold for habitual and fast gait in development of Type 2 diabetes. The deterioration in glucose tolerance in healthy elderly subjects is thought to be derived from a decrease in both insulin secretion and action [18], suggesting that diminished muscle mass leads to insulin resistance. Diminished muscle mass may lead to a lowering of gait speed, walking difficulty and sarcopenia. Our findings suggest that precise assessment of muscle characteristics using gait speed for early detection of sarcopenia and impaired muscle quality is a potential target for medical intervention not only to encourage an appropriate lifestyle but also to prevent development of Type 2 diabetes in elderly persons.
Although there is no consensus regarding the effect of aging on average scores for several of the measurements, such as the 5 BBS markers, average gait speeds for subjects over 60 years of age without known impairments have ranged from 0.60 to $1.45 \mathrm{~m} / \mathrm{s}$ for HGS and from 0.84 to $2.1 \mathrm{~m} / \mathrm{s}$ for FGS [12]. In other reports based on elderly Japanese subjects, FGS was comparable to that in our study, from 6.7 (SD 1.8) to 8.6 (SD 2.6) seconds for a $10 \mathrm{~m}$ walk [14]. In our study, HGS and FGS were 1.48 (SD 0.26) and 1.85 (SD 0.30) m/s, respectively. Accordingly, results from our study are similar. It was difficult to express relationships between diabetes onset and tandem stance or standing on one leg, because 36 participants (35.3\%) censored because of reaching the ceiling performance in both legs. Average TUG score, widely used as an examination of lower extremity function, mobility, and fall risk, varies from 8.5 to 15 seconds in elderly people [12], whereas it was 7.25 (SD 1.36) seconds in our study. In addition, a previous study reported varying maximal step length among Japanese elderly subjects as being from 73.3 (SD 18.1) to 91.2 (SD 24.6) cm [14], whereas our mean maximal step length was around 90 $\mathrm{cm}$ before normalizing to height. To our knowledge, although these discrepancies could have affected the results, no reports have been published that compare TUG score or maximal step length with development of Type 2 diabetes. In addition, although mean grip strength of our subjects was comparable to standard grip strength in elderly Japanese [20], those values were extremely low, nearly one-half, compared with the values in another study that reported the relationship between grip strength and Type 2 diabetes in middle-aged subjects [21]. This may be one reason why we did not detect a relationship between them in our study.

A substantial inverse association was observed only between gait speed and risk of Type 2 diabetes; no association was observed with other measurements. For example, TUG and the 5 measured parameters from BBS themselves are targeted mainly at measuring balance performance, not physical activity. Grip strength is thought to be simply related to muscle strength, but muscle mass itself is an important target for insulin and closely related to insulin sensitivity. Consequently, although correlated with each other, the other parameters may assess different physiological functions. In other words, among well-functioning elderly, performance on measures other than the 2 gait speeds is highly affected by simple muscular strength, sense of balance, and perseverance, which in turn may be less strongly related to diabetes than the functional limitations related to gait speed.

Walking performance has been shown to reflect health and functional statuses of elderly subjects, and in observational studies walking speed is a powerful predictor of 
survival [22] and long-term risk of disability [23]. In addition, cross-sectional and longitudinal analyses in the Health Aging and Body Composition study have demonstrated that older individuals with diabetes have lower muscle strength and muscle quality compared with their counterparts without diabetes [24,25]. A subject's ability to increase or decrease walking speed suggests the potential to adapt to varying environments and task demands. In other words, compared to HGS, which might predict the development of muscle-function impairment, FGS may predict the potential capacity of the strength of muscle, which is an important target organ of insulin. Therefore, FGS was a more sensitive marker for development of Type 2 diabetes than HGS in this study. Indeed, Hu et al. found a strong association between questionnaire-assessed walking pace and risk of Type 2 diabetes, although their study subjects were middle-aged women and methods of diabetes diagnosis differed [5].

Although HbA1c and category of IFG were significant predictive indicators for developing Type 2 diabetes in our study, BMI was not. HbA1c is known to be a risk factor for Type 2 diabetes [26] and HbA1c is an effective screening tool for diabetes diagnosis among the Japanese and other populations $[27,28]$. IFG is also strongly related to glucose metabolism and is widely recognized as a risk factor for Type 2 diabetes [29,30]. On the other hand, BMI might not accurately reflect body composition or obesity, because a significant decline in muscle mass, especially in the legs and arms [31], and body fat accumulation are observed in elderly subjects [32]. Goodpaster et al. reported the possibility that elderly subjects with normal body weight are at risk for Type 2 diabetes when they possess an inordinate amount of muscle fat or visceral abdominal fat [33]. This might explain why BMI was not significant in our study; another reason could be lack of statistical power.

Our study has several potential limitations. First, the association between the development of Type 2 diabetes and physical performance could be merely an epiphenomenon of aging, and therefore further research into the association should be undertaken. Second, other measures of glucose metabolism, such as insulin levels or data from the glucose tolerance test, were absent in our dataset. It is difficult to investigate the potential association of glucose metabolism and other confounders such as waist circumference, the presence or absence of muscle impairment, or walking performance. Third, only 9 subjects developed diabetes during the follow-up period because of the limited sample size and duration of followup. Fourth, diagnostic criteria for Type 2 diabetes were based only on fasting glucose level, so misclassification could have occurred. Lastly, this study utilized an observational design that did not allow us to precisely determine the effect of gait speed on diabetes. In addition, to generalize our conclusions to other cultures with different genetic and environmental factors, further study is needed.

In summary, among 10 performance-based parameters of physical activity, we found a significant inverse association between low physical performance expressed as diminished gait speed and development of Type 2 diabetes. We conclude that there may be a benefit in encouraging physical activity and preserving walking capacity for preventing Type 2 diabetes.

\section{REFERENCES}

[1] Wild, S., Roglic, G., Green, A., Sicree, R. and King, H. (2004) Global prevalence of diabetes: Estimates for the year 2000 and projections for 2030. Diabetes Care, 27, 1047-1053. http://dx.doi.org/10.2337/diacare.27.5.1047

[2] International Diabetes Federation, IDF Database Atlas 5th ed. http://www.idf.porg/diabetesatlas

[3] Nakano, T. and Ito, H. (2007) Epidemiology of diabetes mellitus in old age in Japan. Diabetes Research and Clinical Practice, 77, S76-S81. http://dx.doi.org/10.1016/j.diabres.2007.01.070

[4] Lim, K. and Taylor, L. (2005) Factors associated with physical activity among older people-A population-based study. Preventive Medicine, 40, 33-40. http://dx.doi.org/10.1016/j.ypmed.2004.04.046

[5] Hu, F.B., Sigal, R.J., Rich-Edwards, J.W., et al. (1999) Walking compared with vigorous physical activity and risk of Type 2 diabetes in women: A prospective study. The Journal of the American Medical Association, 282, 1433-1439. http://dx.doi.org/10.1001/jama.282.15.1433

[6] Maejima, H., Sunahori, H., Otani, T., Sakamoto, N., Yoshimura, O. and Tobimatsu, Y. (2009) Effect of long-term, community-based daily exercise on the ability to control the dynamic standing balance of Japanese elderly persons in relation to falls. Nursing \& Health Sciences, 11, 128134. http://dx.doi.org/10.1111/j.1442-2018.2009.00453.x

[7] Seino, Y., Nanjo, K., Tajima, N., et al. (2010) Report of the committee on the classification and diagnositic criteria of diabetes mellitus: The committee of the Japan Diabetes Society on the diagnostic criteria of diabetes mellitus. Journal of Diabetes Investigation, 1, 212-228. http://dx.doi.org/10.1111/j.2040-1124.2010.00074.x

[8] Berg, K., Wood-Dauphinée, S., Williams, J.I. and Gayton, D. (1989) Measuring balance in the elderly: Preliminary development of an instrument. Physiotherapy Canada, 41, 304-311. http://dx.doi.org/10.3138/ptc.41.6.304

[9] Duncan, P.W., Weiner, D.K., Chandler, J. and Studenski, S. (1990) Functional reach: A new clinical measure of balance. Journal of Gerontology, 45, M192-M197. http://dx.doi.org/10.1093/geronj/45.6.M192

[10] Hunt, T.N., Ferrara, M.S., Bornstein, R.A. and Baumgartner, T.A. (2009) The reliability of the modified Balance Error Scoring System. Clinical Journal of Sports Medicine, 19, 471-475. http://dx.doi.org/10.1097/JSM.0b013e3181c12c7b 
[11] Sasaki, H., Kasagi, F., Yamada, M. and Fujita, S. (2007) Grip strength predicts cause-specific mortality in middleaged and elderly persons. American Journal of Medicine, 120, 337-342.

http://dx.doi.org/10.1016/j.amjmed.2006.04.018

[12] Steffen, T.M., Hacker, T.A. and Mollinger, L. (2002) Ageand gender-related test performance in community-dwelling elderly people: Six-Minute Walk Test, Berg Balance Scale, Timed Up \& Go test, and gait speeds. Physical Therapy, 82, 128-137.

[13] Schoene, D., Wu, S.M., Mikolaizak, A.S., et al. (2013) Discriminative ability and predictive validity of the timed up and go test in identifying older people who fall: Systematic review and meta-analysis. Journal of American Geriatric Society, 61, 202-208.

http://dx.doi.org/10.1016/j.amjmed.2006.04.018

[14] Hiura, M., Nemoto, H., Nishisaka, K., Higashi, K. and Katoh, T. (2012) The association between walking ability and falls in elderly Japanese living in the community using a path analysis. Journal of Community Health, 37, 957-962. http://dx.doi.org/10.1007/s10900-011-9531-y

[15] Wang, C.Y., Yeh, C.J. and Hu, M.H. (2011) Mobility-related performance tests to predict mobility disability at 2-year follow-up in community-dwelling older adults. Archives of Gerontology and Geriatrics, 52, 1-4. http://dx.doi.org/10.1016/j.archger.2009.11.001

[16] The Expert Committee on the Diagnosis and Classification of Diabetes Mellitus. (1997) Report of the Expert Committee on the diagnosis and classification of diabetes mellitus. Diabetes Care, 20, 1183-1197.

[17] Schoenfeld, D. (1982) Partial residuals for the proportional hazards regression model. Biometrika, 69, 239-241. http://dx.doi.org/10.1093/biomet/69.1.239

[18] Basu, R., Breda, E., Oberg, AL., et al. (2003) Mechanisms of the age-associated deterioration in glucose Tolerance: Contribution of alterations in insulin secretion, action, and clearance. Diabetes, 52, 1738-1748. http://dx.doi.org/10.2337/diabetes.52.7.1738

[19] Sakurai, T., Iimuro, S., Araki, A., et al. (2010) Age-associated increase in abdominal obesity and insulin resistance, and usefulness of AHA/NHLBI definition of metabolic syndrome for predicting cardiovascular disease in Japanese elderly with Type 2 diabetes mellitus. Gerontology, 56, 141-149. http://dx.doi.org/10.1159/000246970

[20] Fujita, Y., Nakamura, Y., Hiraoka, J., et al. (1995) Physical-strength tests and mortality among visitors to healthpromotion centers in Japan. Journal of Clinical Epidemiology, 48, 1349-1359.

http://dx.doi.org/10.1016/0895-4356(95)00533-1

[21] Wander, P.L., Boyko, E.J., Leonetti, D.L., McNeely, M.J., Kahn, S.E. and Fujimoto, W.Y. (2011) Greater hand-grip strength predicts a lower risk of developing Type 2 diabetes over 10 years in leaner Japanese Americans. Diabetes Research and Clinical Practice, 92, 261-264. http://dx.doi.org/10.1016/j.diabres.2011.01.007

[22] Studenski, S., Perera, S., Patel, K., et al. (2011) Gait speed and survival in older adults. The Journal of the American Medical Association, 305, 50-58. http://dx.doi.org/10.1001/jama.2010.1923

[23] Guralnik, J.M., Ferrucci, L., Simonsick, E.M., Salive, M.E. and Wallace, R.B. (1995) Lower-extremity function in persons over the age of 70 years as a predictor of subsequent disability. New England Journal of Medicine, 332, 556-561.

http://dx.doi.org/10.1056/NEJM199503023320902

[24] Park, S.W., Goodpaster, B.H., Strotmeyer, E.S., et al. (2006) Decreased muscle strength and quality in older adults with Type 2 diabetes: The health, aging, and body composition study. Diabetes, 55, 1813-1818. http://dx.doi.org/10.2337/db05-1183

[25] Park, S.W., Goodpaster, B.H., Strotmeyer, E.S., et al. (2007) Accelerated loss of skeletal muscle strength in older adults with Type 2 diabetes. Diabetes Care, 30, 15071512. http://dx.doi.org/10.2337/dc06-2537

[26] Bennett, C.M., Guo, M. and Dharmage, S.C. (2007) $\mathrm{HbA}(1 \mathrm{c})$ as a screening tool for detection of Type 2 diabetes: a systematic review. Diabet Med, 24, 333-343. http://dx.doi.org/10.1111/j.1464-5491.2007.02106.x

[27] Heianza, Y., Hara, S., Arase, Y., et al. (2011) HbA1c 5.7-6.4\% and impaired fasting plasma glucose for diagnosis of prediabetes and risk of progression to diabetes in Japan (TOPICS 3): A longitudinal cohort study. Lancet, 378, 147-155. http://dx.doi.org/10.1016/S0140-6736(11)60472-8

[28] Nakagami, T., Tominaga, M., Nishimura, R., et al. (2007) Is the measurement of glycated hemoglobin A1c alone an efficient screening test for undiagnosed diabetes? Japan national diabetes survey. Diabetes Research and Clinical Practice, 76, 251-256. http://dx.doi.org/10.1016/j.diabres.2006.09.015

[29] Gabir, M.M., Hanson, R.L., Dabelea, D., et al. (2000) The 1997 American Diabetes Association and 1999 World Health Organization criteria for hyperglycemia in the diagnosis and prediction of diabetes. Diabetes Care, 23, 1108-1112. http://dx.doi.org/10.2337/diacare.23.8.1108

[30] Mukai, N., Doi, Y., Ninomiya, T., et al. (2009) Impact of metabolic syndrome compared with impaired fasting glucose on the development of Type 2 diabetes in a general Japanese population: The Hisayama study. Diabetes Care, 32, 2288-2293.

http://dx.doi.org/10.2337/dc09-0896

[31] Borkan, G.A., Hults, D.E., Gerzof, S.G., Robbins, A.H. and Silbert, CK. (1983) Age changes in body composition revealed by computed tomography. Journal of Gerontology, 38, 673-677. http://dx.doi.org/10.1093/geronj/38.6.673

[32] Raguso, C.A., Kyle, U., Kossovsky, M.P., et al. (2006) A 3-year longitudinal study on body composition changes in the elderly: Role of physical exercise. Clinical Nutrition, 25, 573-580. http://dx.doi.org/10.1016/j.clnu.2005.10.013

[33] Goodpaster, B.H., Krishnaswami, S., Resnick, H., et al. (2003) Association between regional adipose tissue distribution and both Type 2 diabetes and impaired glucose tolerance in elderly men and women. Diabetes Care, 26, 372-379. http://dx.doi.org/10.2337/diacare.26.2.372 